\title{
WHO CRITERIA FOR MEASLES ELIMINATION: A CRITIQUE WITH REFERENCE TO CRITERIA FOR POLIO ELIMINATION
}

\author{
H Kelly (heath.kelly@mh.orgau)1,2, M Riddell ${ }^{1,2}$, A Heywood ${ }^{3,4}$, S Lambert ${ }^{5,6}$ \\ 1. WHO Measles Regional Reference Laboratory, Victorian Infectious Diseases Reference Laboratory, North Melbourne, Australia \\ 2. School of Population Health, University of Melbourne, Australia \\ 3. National Centre for Immunisation Research and Surveillance of Vaccine Preventable Diseases, Sydney, Australia \\ 4. School of Public Health and Community Medicine, University of New South Wales, Australia \\ 5. Queensland Paediatric Infectious Diseases Laboratory, Royal Children's Hospital, Brisbane, Australia \\ 6. Clinical Medical Virology Centre, Sir Albert Sakzewski Virus Research Centre, University of Queensland, Brisbane, Australia
}

This article was published on 17 December 2009.

Citation style for this article: Kelly H, Riddell M, Heywood A, Lambert S. WHO criteria for measles elimination: a critique with reference to criteria for polio elimination. Euro Surveill. 2009;14(50):pii=19445. Available online: http://www.eurosurveillance.org/ViewArticle.aspx?ArticleId=19445

Smallpox was formally declared as eradicated in 1979. Smallpox is the only infectious disease of humans that has ever been eradicated. Poliomyelitis has been eliminated from three of the six World Health Organization (WHO) regions although not all countries within those regions always meet the elimination criteria. Elimination criteria for measles are being discussed. We use poliomyelitis and measles as examples to illustrate our assertion that the current approach to documenting measles elimination relies too heavily on criteria for surveillance quality, disadvantaging countries with long established and relatively inflexible surveillance systems. We propose an alternative approach to documenting measles elimination, with the two key criteria being molecular evidence to confirm the lack of a circulating endemic genotype for at least one year and maintenance of $95 \%$ coverage of one dose of measles-containing vaccine, with an opportunity for a second dose. Elimination status should be reviewed annually. We suggest four principles that should guide development of final criteria to document measles elimination: countries that have eliminated measles should be able to meet the elimination criteria; quality surveillance criteria are necessary but not sufficient to define elimination; quality surveillance criteria should be guided by elimination criteria, not the other way around; and elimination criteria should not differ between the WHO regions without good reason.

\section{Introduction}

Smallpox is the only infectious disease of humans that has been successfully eradicated, with a formal declaration made in December 1979 [1]. At this time, eradication was defined by the World Health Organization (WHO) as the absence of circulating wild virus, manifested as no cases in a defined geographic area for a period of at least three years after cessation of vaccination.

In 1988, the World Health Assembly resolved to eradicate polio globally by the year 2000 . The eradication of poliovirus requires zero cases of poliomyelitis due to wild poliovirus for three years, high quality disease surveillance which meets international standards, and demonstrated capacity of the countries to detect, report and respond to imported polio cases, including those caused by vaccine-derived polioviruses. In addition, laboratory stocks need to be contained and safe management of polio vaccine manufacturing sites assured before the world can be certified as polio-free [2]. Eradication by 2000 was not achieved, but in 2009, polio remained endemic in only four countries. The eradication of polio is now seen as an achievable goal within the next four or five years [3], although some commentators question even this timeline.

More recently, goals for progress towards measles elimination, rather than eradication, have been proposed by a number of WHO regions, including the European and Western Pacific Regions. Member states of the Western Pacific Region, which include Australia, have resolved to eliminate measles by 2012 [4]. The European region aims to eliminate measles by 2010 [5]. Elimination is defined as the sustained interruption of transmission of endemic virus within a defined geographic region. Sustained endemic transmission is defined as an outbreak of more than 100 cases or ongoing transmission with a measles genotype of identical sequence for more than three months [6]. Elimination does not imply that there is no virus within the defined region (this is eradication), but that the transmission of endemic virus has been eliminated [6].

We aim to review the criteria used to define polio eradication and measles elimination in the Australian, European and other international context and discuss alternatives to the criteria for the documentation of the elimination of measles.

\section{Australia and polio}

As a member state of the Western Pacific Region, Australia was declared free of circulating endemic poliovirus only in October 2000 [7], although the last case of endemic poliovirus infection probably occurred around 30 years earlier [8]. The cornerstone of the documentation of polio-free status is surveillance of patients presenting with acute flaccid paralysis (AFP), the most common clinical presentation of acute poliovirus infection, although such cases represent only between one in 100 and one in 1,000 cases of infection [9].

\section{The WHO criteria for adequate AFP surveillance are}

- An annual notification rate of one case presenting with acute flaccid paralysis per 100,000 population aged under 15 years,

- Collection of two stool samples 24 hours apart within 14 days of symptom onset from $80 \%$ of notified cases, 
- Testing of stool samples in a WHO-accredited laboratory to exclude wild poliovirus as the cause of the patient's symptoms [9].

Although countries where polio had been endemic in the recent past have met these criteria, Australia has consistently failed to do

\section{F I G U R E}

Acute flaccid paralysis notification rate per 100,000 population under 15 years of age, Australia 1995-2008



The WHO performance indicator expected rate is $>1$ notification of AFP per 100,000 population aged $<15$ years. so. Of the 14 years that AFP surveillance has been undertaken in Australia, the targets for case ascertainment have been achieved in only five years (Figure) and the criteria for stool collection have never been met [10].

We have previously shown, at least for the state of Victoria and by inference for other Australian states, that it was not a lack of AFP cases that led to notification rates below the WHO target, but incomplete notification of cases [11]. Despite not meeting the WHO AFP surveillance criteria for the maintenance of the documentation of polio-free status, Australia, as a member state of the polio-free Western Pacific Region, is nonetheless acknowledged to have no circulating wild poliovirus.

\section{Australia and measles}

We have previously reviewed the body of evidence to demonstrate that Australia has eliminated the transmission of endemic measles [12]. Although we acknowledged that measles virus was still detected in Australia, we argued that the transmission of endemic measles virus has been eliminated, based on criteria we compiled using the evidence for Australia [12]:

- Absence of an endemic genotype since 1999,

- High proportion of cases imported or linked to an imported case since 1999,

- Containment of outbreaks without the re-establishment of a specific genotype since 1999 ,

T A B L E 1

Australia's documentation of measles elimination compared with the criteria proposed by the WHO Western Pacific Regional Office, September 2007

Western Pacific Regional Office criterion for progress towards measles

\section{elimination}

1. Confirmed measles cases $<1$ per million

2. Reported suspected measles cases $>2$ per 100,000

3. At least $80 \%$ of districts reporting $>1$ per 100,00 suspected cases

4. At least $80 \%$ of cases investigated within 48 hours

5. At least $80 \%$ of cases with adequate blood samples collected

6 . At least $80 \%$ of cases with laboratory results within seven days

7. At least $80 \%$ of clusters with samples for virus isolation

8. Two-dose MCV coverage $>95 \%$

9. At least $80 \%$ of clusters with $<10$ cases

10. Absence of endemic measles virus

\section{Criterion status in Australia}

Met in 2005 and 2007; not met in 2006 or 2008

Not available at a national level; met in the state of Victoria since 1999 Data not collected at a national level

Data not available at a national level

Data not available at a national level

Data not available at a national level

Data not available at a national level

MCV1 >95\% and MCV2 >90\%

Data not available at a national level

No endemic measles virus since 1999

MCV: measles-containing vaccine; WHO: World Health Organization. Adapted from Heywood et al. [12].

T A B L E 2

Alternative criteria for the documentation of measles elimination

\section{Criterion}

The absence of an endemic measles genotype for at least 12 months

One dose MCV coverage $>95 \%$ with the opportunity for a second dose.

\section{Justification}

Based on the criterion by which England and Wales declared the re-establishment of endemic viral transmission [18].

One dose of MCV administered at the age of 12 months with coverage $>95 \%$ was modelled to be more likely to maintain elimination status than a two-dose regime [19]. The failure to maintain high measles vaccine coverage led to measles becoming again endemic in England and Wales [18]. 
- Maintenance of an effective reproductive number for measles $<1$ since 1999,

- Serological evidence of population immunity $>90 \%$ since 2002 ,

- Consistently high two-dose vaccination coverage since 2004: $>95 \%$ for the first dose of measles-containing vaccine (MCV) and $>90 \%$ for the second dose of MCV,

- $<1$ notified confirmed endemic case per million population since 2005.

We examined Australia's ability to meet the criteria proposed by the Western Pacific Regional Office (WPRO) in 2007 for the documentation of progress towards measles elimination in member states of the Western Pacific Region (Table 1) $[12,13]$.

The first WPRO criterion requires a national incidence of less than one confirmed measles case per million population. A confirmed case includes laboratory-confirmed cases, cases epidemiologically linked to a laboratory-confirmed case, or clinically confirmed cases; imported cases are excluded. In Australia, national surveillance data are not adequate to demonstrate the proportion of cases that are imported. In both 2005 and 2007, less than one case per million was reported in Australia, inclusive of imported cases. However, an importation leading to a widespread outbreak in 2006 resulted in a notification rate exceeding six cases per million population. Cases in 2008 also exceeded one case per million population. We are unable to quantify the number of confirmed measles cases in 2006 and 2008 that were not imported or directly related to importation [14].

In the first quarter of 2009, 78 cases of measles were notified in Australia, of which 17 were related to importation [15]. Large outbreaks occurred in Queensland and Victoria and smaller outbreaks occurred in other states. In the three months from January to March alone, the number of indigenous cases exceeded an annual notification rate of one per million inhabitants. However, extensive case follow-up and genotyping confirmed that the outbreaks were due to several different genotypes (D4, D8, D9 and $\mathrm{H} 1$ ) and that no one genotype has been circulating for more than 12 months.

The next six WPRO criteria relate to setting surveillance standards for suspected case investigation. Australia is unable to meet any of these criteria (Table 2). The final three criteria refer to vaccine coverage ( $\geq 95 \%$ two-dose MCV coverage), proving that $80 \%$ of outbreaks have fewer than 10 cases and demonstrating the absence of an endemic measles genotype. Australia meets only the third of these criteria. However, in addition to the WPRO criteria, Australia has demonstrated a measles immunity exceeding $90 \%$ in the population in serological surveys [12], and a number of disease modelling studies have consistently estimated that the reproductive number for measles was less than one in a number of studies from Australia, indicating that endemic measles transmission cannot be sustained [12].

\section{Measles elimination in other countries}

In order of the year of declaration, nine countries - Finland, Cuba, England and Wales, Brazil, Mexico, Canada, the United States (US), South Korea and Australia - have publicly declared measles elimination using a variety of criteria (listed in Table 2 of the paper by Heywood et al. [12]). However, unlike the other countries in this list, the Australian government has not formally ratified the declaration of measles elimination in Australia. The mode and median number of the 10 WPRO criteria that these countries satisfied was two (range: one to eight). South Korea, which satisfied eight of the 10 criteria, and Australia, which satisfied only two, are the only two nations in the Western Pacific Region whose declaration might be constrained by WPRO criteria. Finland, which has remained measles-free for 25 years, reports only the two criteria of low incidence and high vaccine coverage [16].

It is clear that disease elimination cannot be declared in the absence of high quality laboratory-enhanced surveillance. Reflecting this, the WPRO criteria for progress towards measles elimination include a number of specific laboratory indicators for high quality surveillance. In countries such as England and Wales, the US and Australia, specific WHO performance indicators for surveillance are difficult to satisfy. These countries were approaching measles elimination prior to the publication of the WHO elimination criteria, and development of national surveillance systems preceded the smallpox and polio eradication programmes. Collating and summarising surveillance data from different state and local sources at a national level is often difficult. Some developed countries such as the US, did not attempt to justify their poliofree status through AFP surveillance [2]. Surveillance systems in these countries were established outside the WHO framework, and do not have routine mechanisms to capture the surveillance process data specified by the WHO and reflected in the WPRO guidelines for the documentation of the eradication of polio or the elimination of measles. England and Wales declared measles elimination in 2003 prior to the establishment of formal elimination criteria [17] The laboratory-enhanced measles surveillance system of England and Wales does not meet all the surveillance benchmarks specified by WPRO criteria. Despite this, the system rapidly detected the re-establishment of endemic measles in England and Wales in 2008 [18]. Furthermore, the experience of England and Wales demonstrates the critical fact that elimination is an ongoing task. While wild virus is circulating elsewhere, vaccine coverage needs to remain high to prevent the re-establishment of sustained transmission of measles virus.

Reviewing the evidence which England and Wales used to declare elimination before acknowledging the re-establishment of endemic measles transmission illustrates the relative importance of elimination criteria $[17,18]$ Measles elimination was declared in England and Wales using the following evidence [17], with the relevant WPRO criteria in brackets:

- MCV1 coverage of over 90\% until 1998 (WPRO criterion: twodose coverage at least 95\%),

- Average number of measles cases of 1.8 per million inhabitants per year 1995-2001 (WPRO criterion: <1/million/year),

- Small number of large clusters, four clusters with 10-24 cases and four clusters with 25 or more cases (WPRO criterion: $\geq 80 \%$ of outbreaks or transmission foci with $<10$ cases),

- $23 \%$ of sporadic cases and $43 \%$ of clusters linked to a known imported case (no specified WPRO criterion),

- Suspected measles case identification rate ca. 4.4 per 100,000 per year (WPRO criterion: $>2 / 100,000$ ) with $66 \%$ tested (WPRO criterion: $>80 \%$ tested),

- Wide variety of genotypes with absence of previous endemic genotype (WPRO criterion: no endemic genotype),

- Effective measles reproductive number estimated as 0.5-0.7 by a variety of methods (no specified WPRO criterion).

England and Wales, as part of the WHO European region, are not bound by the WPRO criteria for assessing progress towards measles elimination, but other WHO regions are proposing similar criteria. The WPRO criteria are used here to illustrate the comparison 
of evidence for elimination with published criteria for assessing progress towards elimination required in one WHO region. Moreover, it is reasonable to expect that a country that has eliminated measles should satisfy criteria assessing the progress towards elimination. The interim criteria from the WHO Regional Office for Europe that would guide member states in declaring elimination [5] include the following:

- Vaccination coverage: achieving and maintaining at least 95\% coverage with MCV1 and MCV2 in all districts and nationally;

- Outbreak size: At least $80 \%$ of outbreaks should have less than 10 confirmed measles cases;

- Incidence: Achieving a measles incidence of less than one confirmed case per million population per year, excluding cases confirmed as directly imported;

- Endemic measles virus strain(s): zero cases of measles caused by an endemic strain for at least 12 months, i.e. evidence of the absence of endemic transmission by demonstrating zero cases of measles or zero cases with identical genotype sequence over a period of 12 months.

Guidelines for measles elimination criteria in the European region are currently in late draft form, but a recently published review of progress towards measles elimination in Europe confirms the inclusion of the vaccine coverage and measles incidence criteria [5]. A number of surveillance criteria have also been added to the elimination criteria:

- $100 \%$ of member states should report monthly to WHO on measles cases;

- $80 \%$ of member states should submit at least $80 \%$ of casebased reports each month, and submit at least $80 \%$ of reports on time.

When declaring measles elimination in 2003, England and Wales did not satisfy the criteria related to vaccine coverage or measles incidence. In addition, the surveillance criteria were not reported at the time.

\section{Measles elimination criteria: an alternative approach}

The experience of all countries that have eliminated measles highlights a general problem with WHO criteria for progress towards elimination. It is not possible for most countries that have clearly eliminated measles to meet the criteria for progress towards elimination. This is a strange anomaly.

Since elimination criteria are yet to be finalised, we suggest that consideration be given to documenting measles elimination using only two criteria:

- The absence of an endemic measles genotype for at least 12 months,

- One-dose MCV coverage of at least 95\% with an opportunity for a second dose.

In conjunction with suitable surveillance standards, these criteria could also be used for assessing progress towards elimination. Justification for these criteria is presented in Table 2.

Table 3 evaluates the two proposed alternative criteria for measles elimination against evidence presented by the nine countries declaring elimination. All countries reported on measles vaccine coverage targets and all except England and Wales satisfied this criterion. Only Finland and Mexico did not provide evidence of the absence of circulating genotypes, but would without doubt be able to report on these criteria on an annual basis.

Although not absolutely necessary, these criteria could be supported by the demonstration of a reproductive number of less than one for measles and the estimation of at least $90 \%$ population immunity. While low measles notification rates are important, we believe that a number of confirmed cases under one per million is

TA B L E 3

Assessment of alternative criteria for measles elimination by countries declaring measles elimination

\begin{tabular}{|c|c|c|}
\hline \multirow[b]{2}{*}{ Country declaring measles elimination and year of declaration } & \multicolumn{2}{|c|}{ Alternative elimination criteria } \\
\hline & $\begin{array}{l}\text { Absence of an endemic measles genotype for } \\
\text { at least } 12 \text { months }\end{array}$ & $\begin{array}{l}\text { One-dose } \text { MCV }^{*} \text { coverage of at least } 95 \% \\
\text { plus opportunity for second dose }\end{array}$ \\
\hline Finland, 1994 & Not reported & $>97 \%$ two-dose coverage \\
\hline Cuba, 1998 & Reported absence of circulating virus & $\begin{array}{l}\text { One-dose coverage } 98 \% \text { with catch-up } \\
\text { campaigns }\end{array}$ \\
\hline England and Wales, 2003 & Variety of circulating genotypes confirmed & MCV1 coverage >90\%; MCV2 introduced in 1996 \\
\hline Brazil, 2003 & No endemic genotype & $>95 \%$ two-dose coverage since 1997 \\
\hline Mexico, 2004 & Not reported & $\begin{array}{l}>95 \% \text { coverage at age } 1-6 \text { years since } 1996 ; \\
>97 \% \text { coverage at age } 6-10 \text { years since } 1999\end{array}$ \\
\hline United States, 2004 & No endemic genotype & $\begin{array}{l}>90 \% \text { coverage at age } 19-35 \text { months; } 98 \% \\
\text { coverage at school entry; >92\% of school } \\
\text { children immune }\end{array}$ \\
\hline Canada, 2004 & No endemic genotype since 1998 & MCV1 coverage >95\%; MCV2 introduced in 1996 \\
\hline Republic of Korea, 2006 & No endemic genotype & $\begin{array}{l}>95 \% \text { two-dose coverage; } 93 \% \text { of school } \\
\text { children immune }\end{array}$ \\
\hline Australia, 2008 (declaration not endorsed by national a & No endemic genotype since 1999 & MCV1 coverage $>95 \%$ MCV2 coverage $>90 \%$ \\
\hline
\end{tabular}


not a necessary requirement for elimination to be declared, because of residual susceptibility in young adults documented in a number of countries [20-22] and because there is an increased risk of transmission within susceptible groups that may have religious or other objections to vaccination. It is, however, necessary to demonstrate that an importation of a specific measles genotype into a susceptible subgroup does not result in transmission of that measles genotype in the wider population over a period of more than 12 months, as has occurred in England and Wales. In Australia, 22 confirmed cases notified in a year will exceed the threshold of one confirmed case per million. Small outbreaks among young adults resulting from importations have regularly resulted in higher numbers of annual cases during the period when there was no endemic measles genotype [23]. These importations have not led to the re-establishment of endemic measles transmission in Australia.

Surveillance criteria are important for the documentation of the elimination of endemic measles transmission. Using the proposed alternative elimination criteria, it is only critical that cases and clusters are identified and that a suitable specimen is sent to a WHO-accredited laboratory for genotype identification. As already recommended by WHO, all suspected cases of measles should have a serum sample sent to an accredited laboratory for testing measles IgM by a commercial enzyme-linked immunosorbent assay. We further suggest that a suitable specimen for genotyping, preferably a nose/throat swab [24], should be collected from all serologically confirmed cases that are not part of clusters and from a minimum of two cases at the start and two cases at the end of any identified cluster. Placing the emphasis on identifying the absence of an endemic genotype over a 12-month period requires efforts to be focussed on genotype capture, rather than performing individual serological tests within a nominated time. If using the alternative criteria suggested here, it would not be necessary to confirm a case within seven days as is specified in the WPRO criteria. However it would still be necessary to collect a specimen suitable for genotype identification not more than two weeks after rash onset [24]. When countries do not have a national laboratory that is able to perform measles genotyping, appropriate specimens could be referred to a regional laboratory for genotyping, with all results reported to the WHO in order to monitor international transmission patterns [25].

The WPRO criteria related to outbreaks (criteria 7 and 9, Table 1) can be subsumed into the single criterion of complete absence of endemic measles genotype (criterion 10). While it may be difficult to find all cases that are not part of a cluster, all countries with an active surveillance system should be able to recognise clusters. In Finland, where measles has been eliminated for 25 years, it is noted that 'some sporadic imported cases may have escaped our attention, but clusters of secondary cases would almost certainly have been detected had they occurred' [16].

\section{Conclusions}

Despite best intentions and a considerable amount of effort, Australia has not been able to maintain WHO AFP surveillance criteria for the documentation of polio eradication [26]. However, it is accepted that Australia is free of circulating wild poliovirus, the single most important criterion for eradication. We have provided evidence to support our claim that Australia has eliminated measles transmission, but cannot satisfy the criteria for documenting progress towards elimination promulgated by the WHO WPRO. Neither has this evidence resulted in a formal declaration of measles elimination in Australia. Incidentally, we note that the WHO position on the status of measles elimination in Australia is not completely clear. The WHO document Global measles and rubella laboratory network - update published in 2005 [27], prior to presentation of evidence for measles elimination in Australia, acknowledged measles elimination in Australia. Map 1 in that document states that 'Measles has been eliminated from the Western Hemisphere and Australia' [emphasis added] and did not include any countries from the western hemisphere or Australia on the map. The document also noted that multiple genotypes had been detected from imported cases [27]. However, a more recent WHO publication suggests that the Republic of Korea is the first and only country in the Western Pacific Region to have achieved elimination [28].

We believe it is appropriate to separate criteria for the documentation of measles elimination from surveillance performance and laboratory accreditation. We suggest it may be worth considering only two criteria for the documentation of measles elimination with an annual review of elimination status. Finally we suggest there are four principles that should guide the development of formal documentation of measles elimination:

1. Elimination criteria should be able to be met by countries that have eliminated measles;

2. Quality surveillance criteria are necessary but not sufficient to define elimination;

3. Quality surveillance criteria should be guided by elimination criteria, not the other way around;

4. Without good reason, elimination criteria should not differ by WHO region.

\section{Acknowledgements}

B Thorley and K Grant from Australia's National Poliovirus Reference Laboratory kindly provided the Figure. Dr Thorley provided advice on polio eradication in Australia. We also thank D Featherstone, Dr A Dabbagh, Dr D Sniadack and Dr P Strebel, all from WHO, for their critical comments.

\section{Author declaration:}

All authors contributed to the ideas and writing of this manuscript and further declare this manuscript represents the personal opinions of the authors and does not reflect the opinions of their employers.

\section{References}

1. World Health Organization. Fenner F, Henderson DA, Arita I, JeZek Z, Ladnyi ID. Smallpox and its eradication: Chapter 24. The Certification of Eradication: Concepts, Strategy and Tactics. Geneva: WHO; 1988. Available from: http:// whqlibdoc.who.int/smallpox/9241561106_chp24.pdf

2. Smith J, Leke R, Adams A, Tangermann RH. Certification of polio eradication: process and lessons learned. Bull World Health Organ. 2004;82(1):24-30.

3. World Health Organization. [Internet].Overview of Polio Eradication in the WHO African Region. Available from: http://www.afro.who.int/polio/overview. html

4. World Health Organization. Regional Office for the Western Pacific. Fifteenth meeting of the Technical Advisory Group on the Expanded Programme on Immunization and Poliomyelitis eradication in the Western Pacific Region. Beijing, China, 8-10 June 2005. (WP)/ICP/EPI/5.2/001-ARS/2004/GE/10(CHN). 2005 Manila:WHO;2005. Available from: http://www.wpro.who.int/NR/rdonlyres/ A21477FE-161E-45A2-B3C7-6D3DB773968A/O/MTGRPT_TAG15.pdf

5. Centers for Disease Prevention and Control. Progress towards measles elimination - European Region, 2005-2008. MMWR 2009;58(6): 142-5. Available from: http://www.cdc.gov/mmwr/preview/mmwrhtml/mm5806a3.htm

6. World Health Organization. Regional Office for the Western Pacific. Field guidelines for measles elimination. Manila:WHO;2004. Available from: http:// www.wpro.who.int/NR/rdonlyres/0F24B92E-AE2C-4C9B-B73B-E16ACB833C35/0/ FieldGuidelines_for_MeaslesElimination.pdf

7. Adams T. Farewell to polio in the Western Pacific. Bull World Health Organ. 2000;78(12):1375. 
8. Kennett ML, Brussen KA, Wood DJ, van der Avoort HG, Ras A, Kelly HA. Australia's last reported case of wild poliovirus infection. Commun Dis Intell. 1999;23(3):77-9.

9. World Health Organization. Expanded Programme on Immunization. Acute flaccid paralysis (AFP) surveillance: the surveillance strategy for poliomyelitis eradication. Wkly Epidemiol Rec. 1998;73:113-4.

10. Roberts JA, Grant KA, Ibrahim A, Thorley BR. Annual report of the Australian National Poliovirus Reference Laboratory, 2007. Commun Dis Intell. 2008;32(3):308-15.

11. Whitfield K, Kelly H. Using the two-source capture-recapture method to estimate the incidence of acute flaccid paralysis in Victoria, Australia. Bull World Health Organ. 2002;80(11):846-51.

12. Heywood A, Gidding $H$, Riddell M, McIntyre P, Macintyre C, Kelly $H$ Elimination of endemic measles transmission in Australia. Bull World Health Organ. 2009;87(1):64-71. Available from: http://www.who.int/bulletin/ volumes/87/1/07-046375/en/index.html

13. World Health Organization. Western Pacific Regional Office. Monitoring measles Surveillance and Progress Towards Measles Elimination. Manila:WHO;2007. Measles Bulletin. 2007;1(13):1-6. Available from: http://www.wpro.who.int/NR/ rdonlyres/7BE6353C-7D82-4368-A300-57DB3F38148D/0/MeasBulletinIssue13.pdf

14. Australian Government. National Notifiable Diseases Surveillance System. Number of notifications of Measles, received from State and Territory health authorities in the period of 1991 to 2007 and year to date notifications for 2008. Canberra: Department of Health and Ageing; 2008. Available from: http:// www9.health.gov.au/cda/Source/Rpt_3.cfm

15. Martin N, Foxwell AR. Measles status in Australia, and outbreaks in the first quarter of 2009. Commun Dis Intell. 2009;33(2):221-31.

16. Peltola H, Jokinen S, Paunio M, Hovi T, Davidkin I. Measles, mumps, and rubella in Finland: 25 years of a nationwide elimination programme. Lancet Infect Dis. 2008;8(12):796-803.

17. Ramsay ME, Jin L, White J, Litton P, Cohen B, Brown D. The elimination of indigenous measles transmission in England and Wales. J Infect Dis. 2003;187 Suppl 1:S198-207.

18. Editorial team. Measles once again endemic in the United Kingdom. Euro Surveill. 2008;13(27):pii=18919. Available from: http://www.eurosurveillance. org/ViewArticle.aspx?ArticleId $=18919$

19. Wood JG, Gidding HF, Heywood A, Macartney K, McIntyre PB, Macintyre CR. Potential impacts of schedule changes, waning immunity and vaccine uptake on measles elimination in Australia. Vaccine. 2009;27(2):313-8.

20. Ehresmann KR, Crouch N, Henry PM, Hunt JM, Habedank TL, Bowman R, et al. An outbreak of measles among unvaccinated young adults and measles seroprevalence study: implications for measles outbreak control in adult populations. J Infect Dis. 2004;189 Suppl 1:S104-7.

21. Zandotti C, Jeantet D, Lambert F, Waku-Koumou D, Wild F, Freymuth F, et al. Re-emergence of measles among young adults in Marseilles, France. Eur $\mathrm{J}$ Epidemiol. 2004;19(9):891-3.

22. Kelly HA, Gidding HF, Karapanagiotidis T, Leydon JA, Riddell MA. Residual susceptibility to measles among young adults in Victoria, Australia following a national targeted measles-mumps-rubella vaccination campaign. BMC Public Health. 2007;7(1):99.

23. Davidson N, Andrews R, Riddell M, Leydon J, Lynch P. A measles outbreak among young adults in Victoria, February 2001. Commun Dis Intell. 2002;26(2):273-8.

24. Riddell MA, Chibo D, Kelly HA, Catton MG, Birch CJ. Investigation of optimal specimen type and sampling time for detection of measles virus RNA during a measles epidemic. J Clin Microbiol. 2001;39(1):375-6.

25. World Health Organization. Manual for the laboratory diagnosis of measles and rubella virus infection. 2nd ed. WHO: Geneva;2007. Available from: www. who.int/immunization_monitoring/LabManualFinal.pdf

26. Whitfield K, Kelly H. Notification of patients with acute flaccid paralysis since certification of Australia as polio-free. J Paediatr Child Health. 2004;40(8):466-9.

27. World Health Organization. Global measles and rubella laboratory networkupdate. [English, French]. Wkly Epidemiol Rec. 2005;80(44):384-8.

28. Progress towards eliminating measles in Japan, 2008. [French, English]. [No authors listed]. Wkly Epidemiol Rec. 2008;83(39):351-5. 